\title{
The association of new-onset diabetes mellitus and medical therapy for benign prostatic hyperplasia: A population-based study
}

\section{CUA PRIZE ESSAY

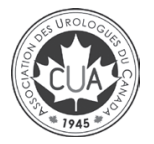

\author{
Jeannette Johnstone, MD'; Avril Lusty, MD',2; Mina Tohidi, MD'; Marlo Whitehead4; Joan Tranmer, PhD, $R N^{4}$; \\ J. Curtis Nickel, MD'; D. Robert Siemens, $M D^{1,5}$
}

'Department of Urology, Queen's University, Kingston, ON, Canada; ${ }^{2}$ Division of Urology, The Ottawa Hospital, Ottawa, ON, Canada; ${ }^{3}$ Department of Surgery, Queen's University, Kingston, ON, Canada; ${ }^{4}$ ICES-Queen's, Queen's University, Kingston, ON, Canada; ${ }^{5}$ Department of Oncology, Queen's University, Kingston, ON, Canada

Cite as: Johnstone J, Lusty A, Tohidi $M$, et al. The association of new-onset diabetes mellitus and medical therapy for benign prostatic hyperplasia: A population-based study. Can Urol Assoc J 2021;15(8):240-6. http://dx.doi.org/10.5489/cuaj.7489

\section{Abstract}

Introduction: Benign prostatic hyperplasia (BPH) and associated lower urinary tract symptoms are highly prevalent in the aging male. Similarly, the prevalence of metabolic syndrome is increasing worldwide, with mounting evidence that these two common conditions share more than age as a predisposing factor. The objective of this study was to determine if medical management of $\mathrm{BPH}$ is associated with an increased risk of new-onset diabetes mellitus (DM) in routine care.

Methods: This population-based, retrospective cohort study expands on a parent study of linked administrative databases identifying patients diagnosed and treated for BPH between 2005 and 2015. The primary outcome of this secondary analysis was a new diagnosis of DM after the index date of $\mathrm{BPH}$ diagnosis. Covariates included age, dyslipidemia, hypertension, and vascular diseases. A Cox proportional hazards regression model was used for inferential statistical analysis.

Results: A total 129223 men were identified with a BPH diagnosis and no prior history of DM. Of those men, 6390 (5\%) were exposed to 5-alpha-reductase inhibitor (5-ARI), 39592 (31\%) exposed to alpha-blocker (AB), and 30545 (24\%) exposed to combination therapy. Compared to those men with no $\mathrm{BPH}$ medication use, those exposed to drugs had an increased risk of new DM. Men treated with combination therapy of $5-\mathrm{ARI}$ and $\mathrm{AB}$ (hazard ratio [HR] 1.30, 95\% confidence interval [CI] 1.25-1.35), 5-ARI monotherapy (HR 1.25, 95\% Cl 1.17-1.34), or AB monotherapy (HR $1.17,95 \% \mathrm{Cl} 1.13-1.22$ ) all were at higher risk of new DM diagnosis after adjusting for important covariates. When calculating the risk of a new diabetes diagnosis measured from the start of drug exposure, men treated with 5-ARIs had an increased risk of DM compared to AB monotherapy as the reference, with HR 1.12 (95\% Cl 1.03-1.21) for 5-ARI monotherapy and HR $1.20(95 \% \mathrm{Cl}$ 1.14-1.25) for combination therapy.

Conclusions: In this large, long-term, retrospective study of men with a BPH diagnosis in routine practice, the risk of a new diagnosis of DM was greater in patients receiving medical management compared to controls. This modest but significant increased risk was highest in men treated with any 5 -ARIs, in combination as well as monotherapy, compared to the ABs.

\section{Introduction}

Benign prostatic hyperplasia (BPH) and associated lower urinary tract symptoms are highly prevalent in the aging male, resulting in a significant public health burden, which globally affects approximately $50 \%$ of men age 65 or older. ${ }^{1,2}$ Similarly, the prevalence of metabolic syndrome, a cluster medical disorder including insulin resistance, obesity, atherogenic dyslipidemia, and hypertension, is increasing worldwide, with almost $50 \%$ of men over age 60 in the United States meeting diagnostic criteria. ${ }^{2,3}$ There is increasing evidence that these two common conditions share more than age as a predisposing factor. ${ }^{4}$ Epidemiological and pre-clinical studies suggest that metabolic syndrome, or its individual components, are associated with the development of urinary symptoms, increase in prostate growth, and progression of $\mathrm{BPH} .{ }^{5,6}$

These previous findings have generated a hypothesis of a causal relationship between metabolic syndrome and the development of $\mathrm{BPH}$, with biological rationale including sex steroid alterations, increased sympathetic tone, and low-grade inflammation. ${ }^{7}$ Perhaps more intriguingly, these patients may share similar metabolic abnormalities of defective insulin-mediated glucose uptake and secondary hyperinsulinemia, with subsequent stimulation of prostate growth by insulin and other related trophic factors. However, this mounting evidence connecting type 2 diabetes mellitus (DM) and risk of BPH is complicated by recent pre-clinical and clinical studies linking the development of DM to the medications used for the management of $\mathrm{BPH}$, specifically the 5 -alpha reductase inhibitors (5-ARI). ${ }^{8-10}$

Both alpha-blockers $(A B)$ and 5-ARIs have been proven to be effective treatment options for $\mathrm{BPH}$ with high-quality evidence $^{2}$ and the use of 5-ARI for BPH has steadily increased. 
Although $5 \alpha$-reductase 2 is mostly expressed in prostate and skin (blocked by finasteride and dutasteride), $5 \alpha$-reductase 1 (blocked by dutasteride) is also active in metabolic tissues, such as the liver and adipose tissue. ${ }^{11}$ Androgens promote normal glucose utilization by stimulating glucose uptake, glycolysis, and mitochondrial oxidative phosphorylation. ${ }^{12}$ Despite large prospective trials of 5-ARIs for BPH not demonstrating any safety signal for $\mathrm{DM},{ }^{13,14}$ a recent non-randomized cohort study suggested that longer-term dutasteride use was associated with an imbalance of metabolic function, lower testosterone levels, increased $\mathrm{HbA} 1 \mathrm{c}$, and altered lipid profiles. ${ }^{9}$ However, larger observational studies investigating the association between BPH medication use and new type 2 DM diagnosis have demonstrated inconsistent results. ${ }^{15,16}$

Although such observational studies are helpful to explore relatively uncommon associations given their large number of men exposed, difficulties in interpretation arise from residual confounding. Indeed, it is problematic controlling for confounding within studies of chronic conditions such as $\mathrm{BPH}$, as there is evidence that certain risk factors are more prevalent among patients starting drug treatment for $\mathrm{BPH}$ as compared to controls. ${ }^{1,17}$ The objective of the present study was to determine if medical management was associated with an increased risk of new-onset DM in males with a $\mathrm{BPH}$ diagnosis and medication use in routine care.

\section{Methods}

This population-based, retrospective cohort study explores the risks of developing subsequent DM after a diagnosis of $\mathrm{BPH}$, specifically exploring the effect of medication use with 5-ARIs and ABs. The present study expands on a parent study (secondary analysis) of linked administrative databases identifying patients diagnosed with $\mathrm{BPH}$ and subsequent diagnosis of congestive heart failure in Ontario, Canada between January 1, 2005 and December 31, 2015. ${ }^{1}$ The maximum followup date was December 31, 2018. Males aged 66 years or older with a diagnosis of $\mathrm{BPH}$ were initially included in the study, as prescription information is routinely captured for those men over 65. International Statistical Classification of Diseases and Related Health Problems (ICD) codes, both ICD-9 and ICD-10, were used to identify the diagnosis of $\mathrm{BPH}$, as the timeline included both versions of ICD classification. Exclusion criteria in the parent study included absence of valid Ontario healthcare, death at index, cardiac failure within the last five years of index date of $\mathrm{BPH}$ diagnosis, and those previously diagnosed with prostate cancer. Patients that used 5-ARI or AB within one year of diagnosis were excluded and, for this secondary analysis, patients with a previous diagnosis of DM were also excluded. The Queen's University Health Sciences and Affiliated Hospitals Research Ethics Board approved this study.

Administrative databases used were all linked through the Institute of Clinical Evaluative Sciences (ICES). Each database is routinely used for research purposes and has been previously validated. ${ }^{18}$ Linked records included those with the Discharge Abstract Database, Ontario Health Insurance Plan (OHIP) physician claims-database, the Ontario Drug Benefit plan, Registered Persons Database and Same Day Surgery database. The accuracy of these databases in terms of quality and coding has been previously discussed. ${ }^{19}$ Statistics Canada's 2016 census data was used to infer socio-economic status (SES) by linking postal code of residence to the mean household income by dissemination area.

The primary outcome of this secondary analysis was a new diagnosis of DM after the index date of BPH diagnosis. In order to account for sampling biases based on baseline risks for DM, men included in the study were then categorized based on their exposure to medical treatment. The control was men who received no treatment, and this was compared to 5-ARI monotherapy, $\mathrm{AB}$ monotherapy, and combination therapy of 5-ARI and AB. 5-ARI included both finasteride and dutasteride, and $A B$ included both selective and nonselective medications. Exposure time to medication (5-ARIs and/or ABs) was listed as number of days of medication use. Covariates that were considered a priori included age, dyslipidemia, hypertension, and vascular disease encompassing previous diagnosis of myocardial infarction, peripheral vascular disease, or cerebral vascular disease. An overall score of comorbidities, using the John Hopkins' Aggregated Diagnosis Groups (ADGs) was used.

Descriptive statistics were used for baseline characteristics describing the cohorts based on medications used for $\mathrm{BPH}$. A Cox proportional hazards regression model was used for inferential statistical analysis. The associated risk of subsequent type $2 \mathrm{DM}$ following the initiation of $\mathrm{BPH}$ medical therapy (5-ARI and $A B$ ) was evaluated using univariate, as well as multivariable competing risk analyses adjusted for all described covariates. Each patient's index date was first diagnosis of BPH. To account for immortal time bias, subsequent analysis included only those prescribed medications, and the risk of DM was then measured from the start of first drug exposure. Statistical significance was set at a two-sided $p$-value of $<0.05$. Data were analyzed using SAS Stat 14.3.

\section{Results}

There were 282462 men included in the study over the age of 66 with a diagnosis of BPH. After exclusions, 129223 men met the study criteria. Of those men included with a $\mathrm{BPH}$ diagnosis, 52696 had no evidence of either $A B$ or 5-ARI prescriptions, whereas $6390(5 \%)$ were exposed to 5-ARI, 39592 (31\%) exposed to AB, and 30545 (24\%) exposed to combination therapy. The median age of the cohort was 73 years and other baseline characteristics between men based on BPH medication use were similar, including past 


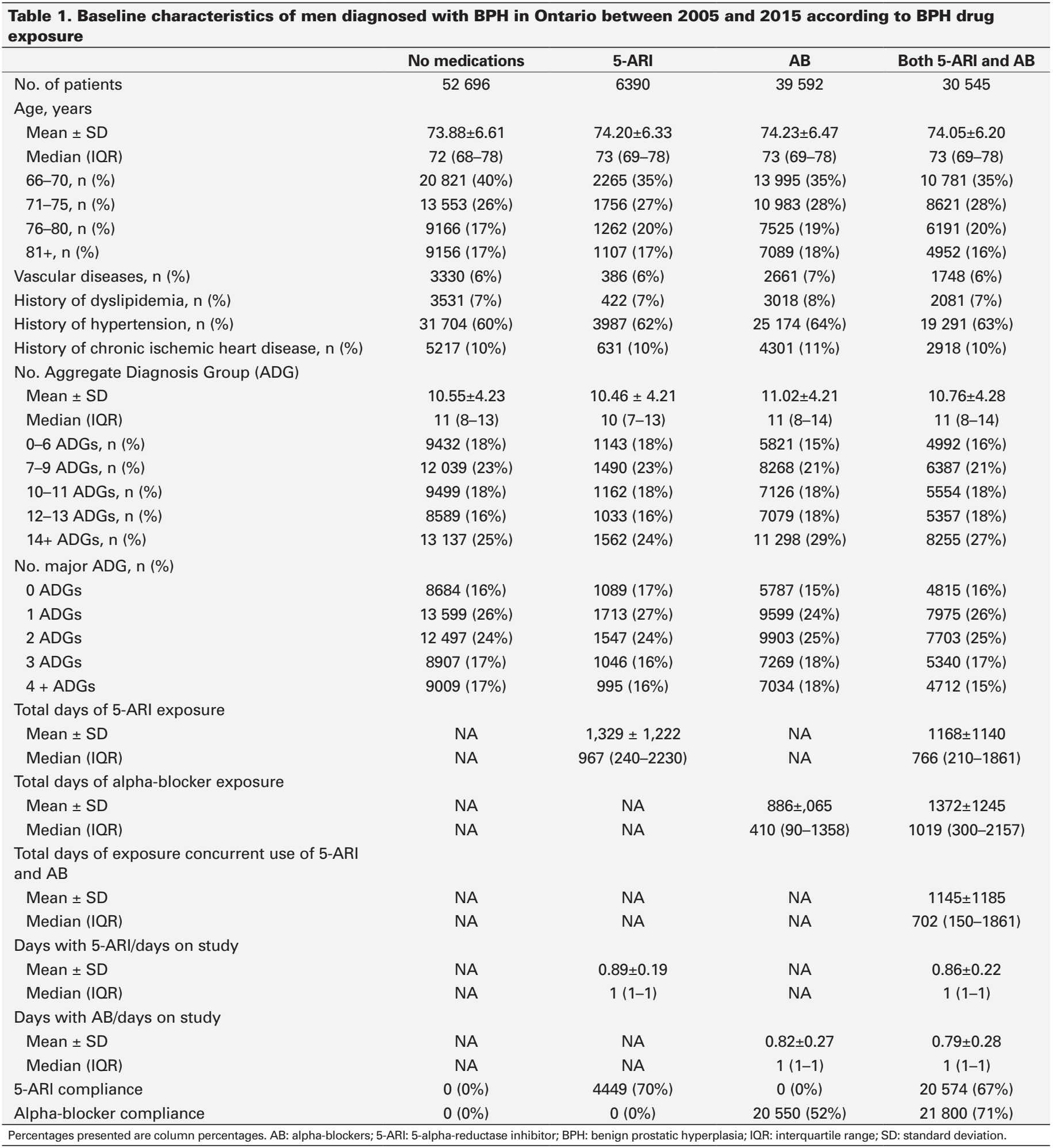

history of cardiac disease, hypertension, and dyslipidemia (Table 1). The total number of days on drug and measures of continuous use are also outlined in Table 1. This demonstrated that those on $\mathrm{AB}$ monotherapy had a median number of 410 days of exposure (90-1358), which was similar to other BPH medication exposure groups.

Men prescribed 5-ARI monotherapy, AB monotherapy, or combination therapy had an increased risk of DM in 
BPH medications associated with new-onset diabetes

\begin{tabular}{|c|c|c|c|c|c|}
\hline & \multirow[b]{2}{*}{ Rate } & \multicolumn{2}{|c|}{ Unadjusted } & \multicolumn{2}{|c|}{ Adjusted } \\
\hline & & HR ( $\pm 95 \% \mathrm{Cl})$ & p & HR ( $\pm 95 \% \mathrm{Cl})$ & p \\
\hline 5-ARI exposure & 14.15 & $1.26(1.17-1.35)$ & $<0.001$ & $1.25(1.17-1.34)$ & $<0.001$ \\
\hline Alpha blocker & 12.92 & 1.19 (1.15-1.24) & & $1.17(1.13-1.22)$ & \\
\hline Both 5-ARI and alpha blocker & 14.83 & $1.32(1.27-1.37)$ & & $1.30(1.25-1.35)$ & \\
\hline No medications & 10.74 & Ref & & Ref & \\
\hline \multicolumn{6}{|l|}{ Age at index date } \\
\hline $66-70$ & 13.92 & Ref & $<0.001$ & Ref & $<0.001$ \\
\hline $71-75$ & 13.49 & $0.95(0.91-0.98)$ & & $0.91(0.87-0.94)$ & \\
\hline $76-80$ & 12.17 & $0.85(0.81-0.88)$ & & $0.78(0.75-0.82)$ & \\
\hline $81+$ & 8.51 & $0.58(0.55-0.61)$ & & $0.53(0.50-0.56)$ & \\
\hline \multicolumn{6}{|l|}{ History of dyslipidemia } \\
\hline No & 12.31 & Ref & $<0.001$ & Ref & $<0.001$ \\
\hline Yes & 15.62 & $1.31(1.24-1.38)$ & & $1.16(1.10-1.24)$ & \\
\hline \multicolumn{6}{|l|}{ History of hypertension } \\
\hline No & 10.00 & Ref & $<0.001$ & Ref & $<0.001$ \\
\hline Yes & 14.10 & $1.45(1.40-1.50)$ & & $1.48(1.43-1.53)$ & \\
\hline \multicolumn{6}{|c|}{ History of chronic ischemic heart disease } \\
\hline No & 12.30 & Ref & $<0.001$ & Ref & $<0.001$ \\
\hline Yes & 14.70 & $1.20(1.15-1.26)$ & & $1.13(1.07-1.19)$ & \\
\hline \multicolumn{6}{|l|}{ \# major ADGs } \\
\hline 0 ADGs & 11.88 & Ref & 0.03 & Ref & 0.24 \\
\hline $1 \mathrm{ADGs}$ & 12.70 & $1.06(1.01-1.11)$ & & $1.03(0.98-1.08)$ & \\
\hline 2 ADGs & 12.62 & $1.04(0.99-1.10)$ & & $1.01(0.96-1.06)$ & \\
\hline 3 ADGs & 13.06 & $1.08(1.02-1.14)$ & & $1.04(0.99-1.10)$ & \\
\hline $4+\mathrm{ADGs}$ & 12.26 & $1.01(0.95-1.07)$ & & $0.99(0.94-1.05)$ & \\
\hline \multicolumn{6}{|l|}{ Socio-economic status } \\
\hline 1-Lowest & 13.79 & Ref & $<0.001$ & Ref & $<0.001$ \\
\hline 2 & 13.20 & $0.95(0.91-1.00)$ & & $0.94(0.90-0.99)$ & \\
\hline 3 & 12.97 & $0.94(0.89-0.99)$ & & $0.93(0.88-0.97)$ & \\
\hline 4 & 12.39 & $0.89(0.85-0.93)$ & & $0.87(0.83-0.92)$ & \\
\hline 5-Highest & 10.89 & $0.77(0.74-0.81)$ & & $0.77(0.73-0.81)$ & \\
\hline
\end{tabular}

Adjusted for all covariates shown in Cox proportional hazards model. Time starts from benign prostatic hyperplasia diagnosis. ADG: Aggregate Diagnosis Group; 5-ARI: 5-alpha-reductase inhibitor; Cl: confidence interval; HR: hazard ratio.

comparison to those with a BPH diagnosis and no exposure to medical therapy. Table 2 represents both unadjusted and adjusted models of variables associated with a new-onset DM. As expected, this multivariable analysis shows age, SES, as well as comorbidities linked with metabolic syndrome, to be associated with the primary outcome. Compared to those men with no history of $\mathrm{BPH}$ medication use, those exposed to $A B$ had an increased risk of new DM, with a hazard ratio (HR) of 1.17 (95\% confidence interval [Cl] 1.13-1.22). Similarly, those exposed to 5-ARI monotherapy (HR 1.25, 95\% Cl 1.17-1.34) and combination therapy (HR 1.30, 95\% $\mathrm{Cl}$ 1.25-1.35) were both at higher risk of a new DM diagnosis after adjusting for important covariates.

To address a possible immortal time bias for those initiating $\mathrm{BPH}$ medications, a multivariable competing risk analysis was performed on only those patients exposed to $\mathrm{BPH}$ medical therapy ( $n=76527$ ) starting at first drug exposure. Men treated with 5-ARIs had an increased risk of DM compared to $A B$ monotherapy as the reference, with HR $1.12(95 \% \mathrm{Cl} 1.03-$ 1.21) for 5 -ARI monotherapy and $\mathrm{HR} 1.20(95 \% \mathrm{Cl} 1.14-1.25)$ for combination therapy (Table 3). The cumulative incidence function estimate was adjusted for age, dyslipidemia, hypertension, chronic ischemic heart disease, SES, and major ADG and further demonstrated the increased risk of new DM with 5-ARI exposure compared to AB monotherapy (Fig. 1).

Subsequent sensitivity analyses were performed examining the different BPH medication types demonstrating no significant difference in the primary outcome between selective and non-selective $A B s$ and no difference between finasteride (type 1 5-ARI) and dutasteride (type 1 and 2 5-ARI) in adjusted analysis (data not shown). Any 5-ARI exposure (monotherapy or combination) had an 18\% increased risk of a new DM diagnosis in adjusted analysis compared to AB monotherapy (HR 1.18, 95\% Cl 1.13-1.24). 


\begin{tabular}{|c|c|c|c|}
\hline & \multirow[b]{2}{*}{ Reference } & \multicolumn{2}{|c|}{ Adjusted model } \\
\hline & & HR ( $\pm 95 \% \mathrm{Cl})$ & $\mathbf{p}$ \\
\hline 5-ARI exposure & Alpha-blocker & $1.12(1.028-1.214)$ & $<0.001$ \\
\hline $\begin{array}{l}\text { Both 5-ARI and alpha } \\
\text { blocker }\end{array}$ & Alpha-blocker & $1.20(1.142-1.253)$ & $<0.001$ \\
\hline $\begin{array}{l}\text { Any 5-ARI } \\
\text { (monotherapy \& } \\
\text { combination) }\end{array}$ & Alpha-blocker & $1.18(1.13-1.24)$ & $<0.001$ \\
\hline $\begin{array}{l}\text { Non-selective alpha- } \\
\text { blocker }\end{array}$ & $\begin{array}{c}\text { Selective } \\
\text { alpha-blocker }\end{array}$ & $0.94(0.85-1.04)$ & 0.23 \\
\hline Dutasteride & Finasteride & $0.97(0.82-1.14)$ & 0.72 \\
\hline \multicolumn{4}{|c|}{$\begin{array}{l}\text { Adjusted for age, dyslipidemia, hypertension, chronic ischemic heart disease, socio- } \\
\text { economic status, major ADG in Cox proportional hazards model. Time starts from first drug } \\
\text { exposure. ADG: Aggregate Disease Group; 5-ARI: 5-alpha-reductase inhibitor; BPH: benign } \\
\text { prostatic hyperplasia; Cl: confidence interval; HR: hazard ratio. }\end{array}$} \\
\hline
\end{tabular}

There was a significant effect of time (unit=one day) on any 5-ARI exposure and a new DM diagnosis when analyzed as a continuous variable with at least four years of followup. Because of concern for the assumption of linearity and a desire to determine if there was any threshold of drug exposure on outcome, we also analyzed drug exposure based on different time cutpoints. There was a trend to a lower risk of new DM in adjusted models when exposure was less than one year (HR 0.89, 95\% Cl 0.78-1.02, $\mathrm{p}=0.09$ ), however, analyses investigating other single cutpoints of drug exposure were uninformative. Tertile analysis demonstrated disparate results in that that shortest tertile of drug exposure (less than 603 days) had a 36\% decreased risk of DM compared to the second tertile (603-2015 days), with a HR $0.64(95 \% \mathrm{Cl} 0.56-0.74)$. However, those exposed to the

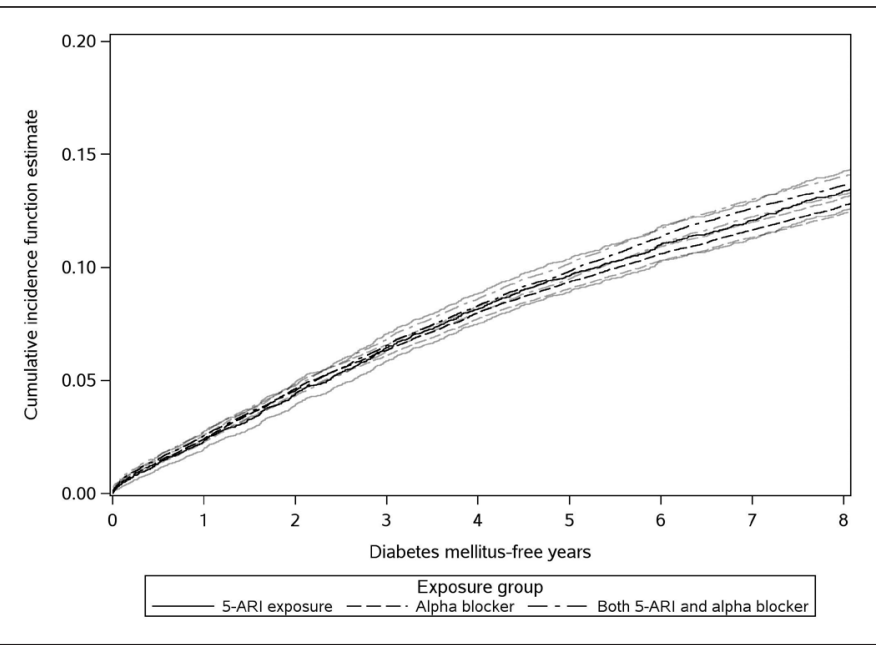

Fig. 1. Cumulative incidence function curve of new diabetes mellitus by exposure group. Adjusted for age, dyslipidemia, hypertension, chronic ischemic heart disease, socio-economic status, and major Aggregated Diagnosis Group. 5-ARI: 5-alpha-reductase inhibitor. drug for longer periods (greater than 2015 days) also had a decreased risk of new DM in adjusted analysis compared to the second tertile, suggesting either a lack of a clear and measurable causal relationship of dose dependence and the primary outcome, bias introduced by drug discontinuation, or residual confounding in those cohorts of men on 5-ARIs for long time periods.

\section{Discussion}

This study used a large, population-based, retrospective cohort representing all men in the province of Ontario with a diagnosis of $\mathrm{BPH}$ in order to determine the potential added burden of commonly prescribed BPH medications, 5-ARI and $A B$, on a new diagnosis of DM. Several interesting and clinically relevant observations were made.

First, we found that exposure to any $\mathrm{BPH}$ medication, 5-ARIs and/or $\mathrm{ABs}$, had a higher rate of new DM diagnosis compared to those men diagnosed with BPH unexposed to these medications. Second, we found that the cumulative incidence of DM was higher in those exposed to 5-ARIs, prescribed either alone or in combination with $\mathrm{ABs}$, compared to $\mathrm{ABs}$ alone. These initial results guided subsequent analysis of the cohort to further interrogate the association of DM with 5-ARIs, which remained significant after controlling for key covariates linked to metabolic syndrome. Third, there was no observed difference between finasteride and dutasteride with the primary outcome in adjusted analysis. Finally, there was some signal associating time on 5-ARIs and DM on adjusted analysis, although there was no well-defined time cutpoint, suggesting some complexity of these associations.

The link between BPH and metabolic syndrome, both its components and associated negative cardiovascular outcomes, is intriguing not only because of their equally high prevalence in the aging male but also some potential shared pathophysiology. ${ }^{5-7}$ Bourke and Griffin were the first to suggest an association between DM and BPH etiology, based on the higher prevalence of the disease among men subjected to prostatectomy than in the general male population..$^{20}$ In 1998, Hammarsten et al published that patients with lower urinary tract symptoms and DM had larger prostate volumes when compared to males without DM. ${ }^{21}$ Increasing evidence lends credence to the concept that the associated hyperglycemia and insulin resistance of those men with DM can significantly increase the risks of $\mathrm{BPH}$ and the subsequent lower urinary tract symptoms. ${ }^{22,23}$ The possible biological mechanisms to bolster these associations include increased sympathetic tone, stimulation of prostate growth by trophic factors, and induction of low-grade oxidative stress and systemic inflammation and, relevant to this study, alterations in sex steroid hormone activity. ${ }^{7}$

Associations have been made between low levels of testosterone and insulin-resistant state, with evidence that sex 
steroid hormones may play a causal role in development of insulin resistance and type 2DM. ${ }^{24-26}$ Interventional studies have shown beneficial effects of exogenous testosterone on components of the metabolic syndrome, type $2 \mathrm{DM}$, and other cardiovascular risk factors, including insulin resistance and high levels of cholesterol. ${ }^{27}$ Testosterone is involved in promoting glucose utilization, modulating expression of insulin receptors, positively influencing key enzymes involved in glycolysis and mitochondrial oxidative phosphorylation. ${ }^{12}$ Given these findings, it is rational to consider the role of BPH medications, specifically the 5-ARIs, on glucose intolerance and DM.

Of the two isozymes of $5 \alpha$-reductase, type 2 predominates in the reproductive tissues, whereas type 1 is also found in the skin, liver, skeletal muscle, and testes. ${ }^{28}$ Finasteride effectively inhibits the type 2 isoenzyme, reducing circulating serum concentrations of DHT by approximately $70 \%$, with transient increases in circulating testosterone; dutasteride, as a dual inhibitor, causes greater reduction in serum DHT concentrations, although it is less clear whether this results in important effects on other androgen-responsive tissues. Murine studies have shown 5-ARIs can increase susceptibility to diet-induced obesity, impaired glucose tolerance, and increased incidence of fatty liver. ${ }^{10}$ Traish et al recently reported increased blood glucose and glycated hemoglobin A1c after several years of dutasteride treatment. ${ }^{9}$

Large, population-based, epidemiological studies have the potential to be adjunctive to prospective clinical trials and single- or multi-institutional observational studies, given their ability to identify and quantify uncommon outcomes, such as adverse effects, in the general population by avoiding various selection biases. Two previous population-level studies investigating the effect of 5-ARI use and new DM were conflicting. ${ }^{15,16}$ Lee et al, using a national research database in Taiwan, reported a lower risk of type 2 DM in men receiving at least 28 days of 5-ARI, although the control group was not well-delineated and the total number of men exposed was low with less fulsome baseline characteristics. ${ }^{15}$ On the other hand, a more robust study using the U.K. Clinical Practice Research Datalink evaluating 55275 men receiving $\mathrm{BPH}$ medications did show an increased risk of 5-ARI exposure compared to tamsulosin. ${ }^{16}$ Although the authors hypothesized that the association would be greatest for dutasteride, given its dual inhibition of 5-alpha reductase, both dutasteride and finasteride had higher risks of subsequent DM: HR 1.32 (95\% Cl 1.08-1.61) and HR 1.26 (95\% Cl 1.10-1.45), respectively. Although the U.K. dataset contains longitudinal records of more than 500 primary care offices, it only accounts for $7 \%$ of the U.K. population and may not be generalizable to the whole population. As well, Wei et al use tamsulosin use as the control group, which could bias the results, as $A B s$ have been shown to improve fasting plasma glucose, insulin, and low-density lipoprotein cholesterol levels. ${ }^{29}$ Finally, the interactions between the ABs and 5-ARIs were not robust, given the low number of men on combination therapy. ${ }^{16}$

This present study incrementally adds to this concept and mirrors many of the findings of the U.K. observational study, with similar effect size of the 5-ARIs on subsequent DM. Importantly, we demonstrate that men who have received medical therapy with either $\mathrm{AB}$ or 5-ARI are at higher risk of new-onset DM compared to a control group that has never been exposed. This finding recapitulates previous observations that certain risk factors of metabolic syndrome or cardiovascular disease are more prevalent among patients starting drug treatment for $\mathrm{BPH}$ as compared to controls. ${ }^{1}$ Also, based on the cumulative incidence function estimates, ABs do not appear to mitigate any increased association of 5-ARI on later DM diagnosis for men on combination therapy.

It is important, however, to consider the current limitations of this study, which include all those of similar populationbased investigations, including its retrospective nature and the accuracy of available diagnostic administrative codes. Another limitation of the study is that the population was predominantly Caucasian and certain baseline characteristics, including body mass index and other prostate-specific data (such as size and prostate-specific antigen), were not available and limited our ability to conduct an instrumental variable analysis to explore unmeasured confounders. Furthermore, it is not possible to infer the compliance of $\mathrm{BPH}$ medication use as compared to drugs dispensed. Finally, the results exploring the biological plausibility of any association with DM with duration of exposure of 5-ARIs was inconsistent.

\section{Conclusions}

In this large, long-term, retrospective, population-based study of men with a BPH diagnosis, the risk of a new diagnosis of DM was greater in patients receiving medical management compared to controls. This modest but significant increased risk was highest in men treated with any 5-ARIs, in combination and as monotherapy, compared to the ABs. As both $\mathrm{BPH}$ and the metabolic syndrome increase with age, it is important to consider this potential increased risk when prescribing these drugs in the context of other risk factors for DM.

Competing interests: The authors do not report any competing personal or financial interests related to this work.

Funding: This study was supported by the Institute for Clinical Evaluative Sciences (ICES), which is funded by an annual grant from the Ontario Ministry of Health and Long-Term Care (MOHLTC). Parts of this material are based on data and information compiled and provided by CIHI. The opinions, results, and conclusions reported in this paper are those of the authors and are independent from the funding sources and CIHI. No endorsement by ICES or the Ontario MOHLTC is intended or should 
Johnstone et al

be inferred. Dr. Siemens had full access to all the data in the study and takes responsibility for the integrity of the data and the accuracy of the data analysis.

\section{References}

1. Lusty A, Siemens DR, Tohidi $M$, et al. Cardiac failure associated with medical therapy of benign prostatic hyperplasia: A population-based study. J Urology 2021;205:1430-7. https://doi.org/10.1097/ JU.0000000000001561

2. Nickel JC, Aaron L, Barkin J et al. Canadian Urological Association guideline on male lower urinary tract symptoms/benign prostatic hyperplasia (MLUTS/BPH): 2018 update. Can Uro Assoc J 2018;12:303-12. https://doi.org/10.5489/cuai.5616

3. Stamatiou K, Lardas M, Kostakos E, et al. The impact of diabetes type 2 in the pathogenesis of benign prostatic hyperplasia: A review. Adv Urol 2009;09:3. https://doi.org/10.1155/2009/818965

4. Hirode G, Wong RJ. Trends in the prevalence of metabolic syndrome in the United States, 2011-2016. JAMA 2020;323:2526-8. https://doi.org/10.1001/jama.2020.4501

5. DiBello JR, loannou C, Rees J, et al. Prevalence of metabolic syndrome and its components among men with and without clinical benign prostatic hyperplasia: A large, cross-sectional, UK epidemiological study. BJU Int 2016;117:801-8. hitps://doi.org/10.1111/bju.13334

6. Vignozzi L, Gacci M, Maggi M. Lower urinary tract symptoms, benign prostatic hyperplasia and metabolic syndrome. Nat Rev Urol 2016;108-19. https://doi.org/10.1038/nrurol.2015.301

7. Breyer BN, Sarma AV. Hyperglycemia and insulin resistance and the risk of BPH/LUTS: An update of recent literature. Curr Urol Rep 2014;462. https://doi.org/10.1007/s11934-014-0462-x

8. Hazlehurst JM, Oprescu Al, Nikolaou N et al. Dual-5 $\alpha$-reductase inhibition promotes hepatic lipid accumuIation in man. J Clin Endocrinol Metab 2016;101:103-13. https://doi.org/10.1210/ic.2015-2928

9. Traish A, Haider KS, Doros $\mathrm{G}$, et al. Long-term dutasteride therapy in men with benign prostatic hyperplasia alters glucose and lipid profiles and increases severity of erectile dysfunction. Horm Mol Biol Clin Investing 2017;30:1-16. htrps://doi.org/10.1515/hmbci-2017-0015

10. Livingstone DE, Barat $P$, Di Rollo EM, et al. 5a-reductase type 1 deficiency or inhibition predisposes to insulin resistance, hepatic steatosis, and liver fibrosis in rodents. Diabetes 2015;64:447-58. https://doi.org/10.2337/db14-0249

11. Upreti $R$, Hughes $K A$, Livingstone $D E$, et al. $5 \alpha$-reductase type 1 modulates insulin sensitivity in men. J Clin Endocrinol Metab 2014;99:1397-406. https://doi.org/10.1210/ic.2014-1395

12. Rao PM, Kelly DM, Jones TH. Testosterone and insulin resistance in the metabolic syndrome and T2DM in men. Nat Rev Endocrinol 2013;9:479-93. https://doi.org/10.1038/nrendo.2013.122

13. Schulman C, Pommerville P, Höfner K, et al. Long-term therapy with the dual 5-alpha-reductase inhibitor dutasteride is well-tolerated in men with symptomatic benign prostatic hyperplasia. BJU Int 2006;97:73-9, discussion 79-80. https://doi.org/10.1111/j.1464-410X.2005.05909.x

14. Andriole GL, Kirby R. Safety and tolerability of the dual 5-alphareductase inhibitor dutasteride in the treatment of benign prostatic hyperplasia. Eur Urol 2003;44:82-8. https://doi.org/10.1016/S03022838(03)00198-2
15. Lee $S$, Yang $Y$, Tsai T, et al. 5-alpha-reductase inhibitors and the risk of diabetes mellitus: A nationwide population-based study. Prostate 2016;76: 41-7. https://doi.org/10.1002/pros.23097

16. Wei L, Lai EC, Kao-Yang YH, et al. Incidence of type 2 diabetes mellitus in men receiving steroid 5a-reductase inhibitors: Population-based cohort. BMJ 2019; 365:11204. https://doi.org/10.1136/bmi.l1204

17. Souverein PC, Herings RMC, De La Rosette JJMCH, et al. Evaluating adverse cardiovascular effects of drug treatment for benign prostatic hyperplasia (BPH): Methodological considerations. I Clin Epidemiol 2001;54:518. https://doi.org/10.1016/S0895-4356(00)00327-9

18. Goel V, Williams JI, Anderson GM, et al. Patterns of healthcare in Ontario. The ICES Practice Atlas 2nd Edition. Ottawa: The Canadian Medical Association; 1996.

19. Hosier GW, McGregor T, Beiko D, et al. Persistent opioid use among patients with urolithiasis: A populationbased study. Eur Urol Focus 2020;6:745-51. https://doi.org/10.1016/i.euf.2019.08.011

20. Bourke JB, Griffin JP. Hypertension, diabetes mellitus, and blood groups in benign prostatic hypertrophy. BJUl 1966;38:18-23. https://doi.org/10.1111/i.1464-410X.1966.tb09675.x

21. Hammarsten J, Hogstedt B, Holthuis N, et al. Components of the metabolic syndrome risk factors for the development of benign prostatic hyperplasia. Prostate Cancer Prost Dis 1998;1:157-62. https://doi.org/10.1038/si.pcan.4500221

22. Kim WT, Yun SJ, Choi YD, et al. Prostate size correlates with fasting blood glucose in non-diabetic benign prostatic hyperplasia patients with normal testosterone levels. J Korean Med Sci 2011;26:1214-8. https://doi.org/10.3346/ikms.2011.26.9.1214

23. Qu X, Huang Z, Meng X, et al. Prostate volume correlates with diabetes in elderly benign prostatic hyperplasia patients. Int Urol Nephrol 2014;46:499-504. https://doi.org/10.1007/s1 1255-013-0555-3

24. Basaria S, Muller DC, Carducci MA, et al. Hyperglycemia and insulin resistance in men with prostate carcinoma who receive androgen deprivation therapy. Cancer 2006;106:581-8. https://doi.org/10.1002/ cncr.21642

25. Jones TH. Testosterone deficiency: A risk factor for cardiovascular disease. Trends Endocrinol 2010;21:496503. https://doi.org/10.1016/i.tem.2010.03.002

26. Oh JY, Barrett-Connor E, Wedick NM, et al. Endogenous sex hormones and the development of type 2 diabetes in older men and women: The Rancho Bernardo study. Diabetes Care 2002;25:55-60. https://doi.org/10.2337/diacare.25.1.55

27. Selvin E, Feinleib M, Zhang $L$, et al. Androgens and diabetes in men: Results from the Third National Health and Nutrition Examination Survey (NHANES III). Diabetes Care 2007;30:234-8. https://doi.org/10.2337/dc06-1579

28. Thigpen AE, Silver RI, Guileyardo JM, et al. Tissue distribution and ontogeny of steroid 5-alpha-reductase isozyme expression. J Clin Invest 1993;92:903-10. https://doi.org/10.1172/JCI116665

29. Pessina AC, Ciccariello L, Perrone F, et al. Clinical efficacy and tolerability of alpha-blocker doxazosin as add-on therapy in patients with hypertension and impaired glucose metabolism. Nutr Metab Cardiovasc Dis 2006;16:137-47. https://doi.org/10.1016/i.numedd.2005.04.005

Correspondence: Dr. D. Robert Siemens, Department of Urology, Queen's University, Kingston, ON, Canada; robert.siemens@kingstonhsc.ca 